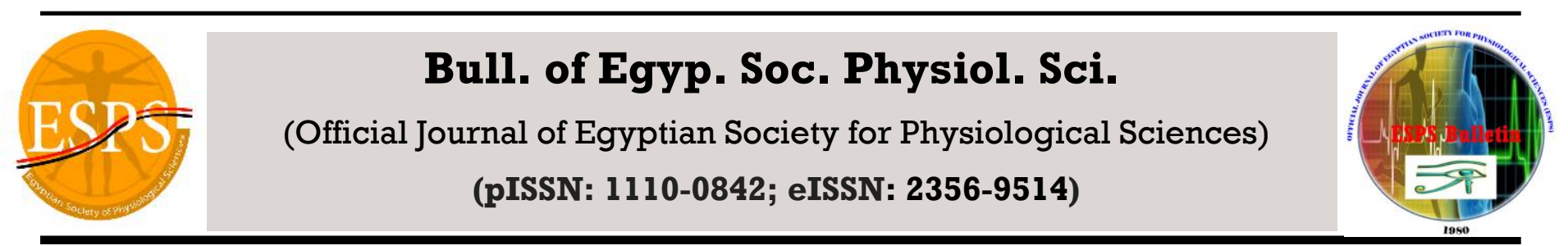

\title{
Remedial Effects of Resveratrol on Chronic Unpredictable Stress-Induced Gastric Lesions in Male Albino Rats
}

\author{
Ismaeel Bin-Jaliah \\ Department of Physiology, College of Medicine, King Khalid University, Abha, Saudi Arabia
}

Received: 4 Nov, 2015

Accepted: 18 Dec 2015

Available online: $25 \operatorname{Dec} 2015$

\section{Keywords}

- Resveratrol,

- Chronic stress,

- Stomach,

- Antiapoptosis,

- Antioxidant.

\section{Abstract}

Objectives: Stress of various patterns has been linked to pathologies of body organs, including gastric mucosal damage. The polyphenol Resveratrol (RES) has been reported to have anti-ulcer properties against some models of gastric ulcer. However, its effect against experimental chronic unpredictable stress (CUS)-induced ulcer per se was not studied before. This study aims to investigate the gastric mucosal damage induced by CUS, its potential prevention by RES, and the mechanisms of action implicated. Methods. Thirty two rats were randomized into 4 groups, 8 rats each: Control, Control+ RES, CUS, and CUS+RES. The CUS was applied for 3 weeks, during which the vehicle (Normal Saline) or RES (25 mg. $\mathrm{kg}^{-1}$ ) was intraperitoneally administered on daily doses. Ulceration index, oxidative, inflammatory and apoptotic parameters were measured. Results. CUS induced severe gastric lesions of various extents, with considerable erosive microscopic features. There was significant increase in the levels of TBARS, TNF- $\alpha$, IL-6, Bax and caspase-3 with concomitant significant decrease in the levels of SOD, GPX, PGE2, and Bcl-2 in CUS rats compared to control rats. Resveratrol treatment to CUS stressed rats brought all of these parameters to control levels, and remarkably maintained the normal morphology and microscopic architectures of gastric tissue. Conclusion. The present data show that supplementation with resveratrol ameliorates CUS-induced gastric lesions via its antioxidant, anti-inflammatory and antiapoptotic potentials; a promising protective consequence that may demand further exploration. 


\section{INTRODUCTION}

The role of stressful life events in the aetiology of various human diseases has been a fertile field of research during the last three decades ${ }^{1}$. It has been increasingly recognized that stress is one of the important components of several diseases including hypertension and coronary heart disease ${ }^{2}$, immunosuppression ${ }^{3}$, metabolic disorders ${ }^{4}$, reproductive dysfunction ${ }^{5}$, mental depression, anxiety, loss of memory, and a host of other organ injury diseases ${ }^{6}$.

Several studies have reported that stomach is one of the main targets of repeated chronic stress ${ }^{1}$. Indeed, different stress-related mucosal pathologies have been described in patients, including gastric mucosal damage, ulceration, and bleeding and were responsible for considerable morbidity and mortality in critically ill patients ${ }^{7,8}$. In animal models, a lot of research has examined the effect of stress on the upper gastrointestinal tract and enough evidences are there for symptoms with chronic stress induced peptic lesions 9 . However, the exact causes for stress-induced ulcers are poorly understood ${ }^{10}$. Several reports implicated the possible roles of reactive oxygen species (ROS) and lipid peroxdation 11, 12, and enhanced production of inflammatory mediators ${ }^{10}$. Also, the pro-inflammatory cytokines have been identified to activate intrinsic apoptotic pathway via induction of the apoptosis executioner protein, caspase- 3 and inhibition of the anti-apoptotioc protein, Bcl2; ultimately resulting in gastric injury and apoptosis ${ }^{13}$.

In experimental medicine and in order to mimic chronic stressful experiences faced by human beings in day-to-day life, chronic unpredictable stress (CUS) paradigm, with varied types and timing of stressors, has been classically used in small animals to induce gastric ulcer ${ }^{11}$. Indeed, it was reported that CUS elevated gastric mucosal oxidative stress, lipid peroxides, tumor necrosis factor- $\alpha$, and caspase- 3 activity $12,14$. However, in the treatment of gastric ulcer of various types, it has been reported that the long term use of the most of the anti-ulcer drugs available on counters leads to various adverse side effects and there is still no complete harmless cure for this disease ${ }^{15}$. Actually, during the past few decades, a widespread search has been launched to identify new anti-ulcer therapies from natural sources, especially those derived from plants ${ }^{16}$.

Resveratrol (RES) is a plant-derived polyphenol (3,5,4'-trihydroxy-trans-stilbene) that is synthesized by a wide diversity of plants, including grapes, raspberries, mulberries, pistachios and peanuts, in response to stress, injury, ultraviolet irradiation and fungal infection as part of their defence mechanism ${ }^{17}$. Resveratrol biological functions include antioxidant, antiinflammatory and anti-cancer effects 18, 19, 20, 21 . Interestingly, RES was shown to protect gastric tissue against the oxidative stress in cholestatic rats $^{22}$.

Taken in consideration the promising antiulcer activity of RES, this study aimed at investigating whether RES exhibits any protective effects against CUS-induced gastric lesions in rats, and to further investigate the mechanisms by which it exerts such effects considering oxidative stress, lipid peroxidation, inflammatory mediators and key markers of apoptotic pathways. 


\section{MATERIALS AND METHODS}

\section{Drugs and chemicals}

Resveratrol is only commercially available as the trans-isomer (trans-resveratrol), and the stable and pharmacologically active form of RES was purchased from Sigma-Aldrich (St. Louis, MO, USA). RES was prepared by dissolving in a saline solution $\quad\left(\begin{array}{llll}0.9 & \% & \mathrm{NaCl}\end{array}\right)$ of $20 \%$ hydroxypropylcyclodextrin (American MaizeProducts Co., Hammond, IN, USA) to the desired final volume used in the experimental procedure. Assay kits for determination of thiobarbituric acid reactive substances levels (TBARS, Cat. No. 10009055) and for determination of superoxide dismutase (SOD, Cat. No. 706002) and gluthathion peroxidise (GPx, Cat. No. 703102) activities were purchased from Cayman Chemical (Ann Arbor, MI, USA). Enzyme-linked immunosorbent assay (ELISA) kits for determination the levels of caspase 3 (Cat. No. R5814), inducible nitric oxide synthase (iNOS, Cat. No R6663), and Bcl-2 (Cat. No. R6813) were purchased from STZ ELISA company, USA. ELISA kit for determination the levels of interleukin 6 (IL-6, Cat. No. ELR-IL6-001) was purchased from RayBio, MO, USA. ELISA kits for determination levels of tumor necrosis factoralpha (TNF- $\alpha$, Cat. No. ab46070), and prostaglandine E2 (PGE2, Cat. No. ab133021) were purchased from Abcam, Cambridge, MA, USA.

\section{Animals}

Thirty two adult male Wistar that are 8 weeks of age and weighing $230 \pm 10 \mathrm{~g}$ were used for the experiments. The animals were obtained from the animal house of the College of Medicine,
King Khalid University, Abha, Saudi Arabia where they were fed standard rat pellets and allowed free access to water before the experiment. They were housed at a controlled ambient temperature of $23 \pm 2{ }^{\circ} \mathrm{C}$ and $50 \pm 10 \%$ relative humidity, with 12-h light/12-h dark cycles. Experiments were performed with the approval of the Biomedical Research Ethics Committee at King Khalid University, Abha, Saudi Arabia (REC. No. 2014-06-09), and all procedures were performed according to the Guide for the Care and Use of Laboratory Animals ${ }^{23}$.

\section{Experimental approach}

After one week adaptation period, rats were randomly assigned to 4 groups, 8 rats each, and were distributed in their corresponding cages (4 per cage, 2 cages for each group) and treated as follows: The first group is the control group (Control) that received normal saline containing $20 \%$ hydroxypropylcyclodextrin $\left(1 \mathrm{ml} . \mathrm{kg}^{-1}\right.$ i.p. $)$ as vehicle. The second group is the resveratroltreated control group (Control+RES) that received RES (at a dose of $25 \mathrm{mg} \cdot \mathrm{kg}^{-1}$ i.p.). The third group is the Chronic unpredictable stress (CUS) group that were exposed to CUS protocol (as detailed below) and received normal saline containing $20 \%$ hydroxypropylcyclodextrin (1 ml.kg ${ }^{-1}$ i.p.). The fourth group is the (CUS+RES) group that were exposed to CUS (as detailed below) with a concomitant daily dose of RES ( $25 \mathrm{mg} . \mathrm{kg}^{-1}$ i.p.). The CUS procedure and RES treatments were carried out for consecutive 21 days on daily dose in the rat groups, as appropriate. The dose selected for RES was based on previous studies that demonstrated safely its antioxidant and anti- 
apoptotic consequences in various disease conditions ${ }^{24}$.

\section{Unpredictable chronic stress (CUS) protocol}

This protocol was applied to the third (CUS) and the fourth (CUS+RES) groups of rats. A set of recognized chronic unpredictable mild stressors were used to induce gastric lesions in rats ${ }^{25}$. Those stressors include: Cage tilting and damp sawdust for $24 \mathrm{~h}(200 \mathrm{ml}$ of water per individual cage, which is enough to make the sawdust bedding wet), Noises for $1 \mathrm{~h}$ (alternative periods of $60 \mathrm{dBA}$ noise for $10 \mathrm{~min}$ and $10 \mathrm{~min}$ of silence), Swimming in $4{ }^{\circ} \mathrm{C}$ cold water for 5 min, Exposure to an experimental room heat at $50{ }^{\circ} \mathrm{C}$ for $5 \mathrm{~min}$, Food deprivation for $48 \mathrm{~h}$, Water deprivation for 24 h, Tail clamp for $1 \mathrm{~min}$, Unpredictable shocks (15 MA, one shock/5 s, $10 \mathrm{~s}$ duration), and Restricted movement for $4 \mathrm{~h}$. One stressor was applied per day, and the whole chronic stress procedure lasted for 3 weeks with a completely random order. On the other hand, the Control and the (Control+RES) groups were housed undisturbed in another experiment room under the standard conditions.

\section{Assessment of gastric tissue lesions}

Six hours after the last treatment on day 21 of the experimental protocol, the rats were humanely sacrificed, under diethyl ether anaesthesia, by decapitation. Each stomach was surgically removed and opened along the greater curvature, and the gastric juice was collected for determination of $\mathrm{pH}$ and gastric acidity. Then, each stomach was directly washed with ice-cold saline and examined for macroscopic mucosal lesions. The gastric mucosal damage was expressed in terms of ulcer index (UI) according to an established equation ${ }^{26,27}$. It depends on the calculation of a lesion index by using of a 0 -to-3 scoring system based on the severity of each lesion. The severity factor was defined according to the length of the lesions: severity factor $0=$ no lesions, severity factor $1=$ lesions $<1 \mathrm{~mm}$ length, severity factor $2=$ lesions $1-4 \mathrm{~mm}$ length, and severity factor $3=$ lesions $>4 \mathrm{~mm}$ length. The lesions score for each rat was calculated as the number of lesions in the rat multiplied by their respective severity factor. The UI for each group was taken as the mean lesion score of all the rats in that group. The percentage of protection by resveratrol was calculated using this equation ${ }^{28}$ : The percentage of protection $=(($ the CUS group UI - the resveratrol treated CUS group UI) / (the CUS group UI)) x 100.

\section{Analysis of gastric juice}

Gastric juice collected from each animal was centrifuged at $3000 \mathrm{rpm}$ for $10 \mathrm{~min}$ to remove any solid debris. Samples of gastric juice were analyzed for hydrogen ion concentration by $\mathrm{pH}$ titration with $0.1 \mathrm{~N} \mathrm{NaOH}$ solutions ${ }^{29,30}$.

\section{Histopathological studies}

Specimens from gastric tissues $(5 \mu \mathrm{m}$ thickness) from all experimental groups were fixed in $10 \%$ neutral buffered formalin, dehydrated in ascending concentrations of ethylalcohol (70-100 $\%$ ), and then prepared using standard procedures for Hematoxylin and Eosin staining, before being evaluated using light microscope. 


\section{Gastric homogenates preparation and biochemical analysis}

Parts of the stomachs obtained from the rats in all groups were freshly washed with phosphate buffered saline (PBS), $\mathrm{pH}$ 7.4. Then they were homogenized with an ultrasonic homogenizer in cold phosphate buffer, $\mathrm{pH}$ 7.4, containing ethylenediaminetetraacetic acid (EDTA). The supernatant obtained was distributed in separate tubes and stored at $-70{ }^{\circ} \mathrm{C}$ for later determination of the levels of: the anti-apoptotioc protein $\mathrm{Bcl}-2$, the apoptosis executioner protein caspase 3, prostaglandin E2 (PGE2), inducible nitric oxide synthase (iNOS), nitrate, nitrite, interleukin 6 (IL6), tumor necrosis factor- alpha (TNF- $\alpha$ ), and thiobarbituric acid reactive substances (TBARS), and the activities of superoxide dismutase (SOD) and superoxide dismutase (GPx); appropriately according to manufacturer's instructions.

\section{RNA extraction and RT-PCR}

Established published sequences of PCR primers used for the detection of Bax, $\mathrm{p} 53$ and $\beta$ actin were used ${ }^{31,32}$. Total RNA was extracted from the frozen parts of gastric tissue $(30 \mathrm{mg}$ ) using an RNeasy Mini Kit (Qiagen Pty. Ltd., Victoria, Australia) according to manufacturer's directions. The concentration of total RNA was measured by absorbance at $260 \mathrm{~nm}$ using a UV1240 spectrophotometer (Shimadzu, Kyoto, Japan). The purity was estimated by the $260 / 280$ $\mathrm{nm}$ absorbance ratio. Single-strand cDNA synthesis was performed as follows: $30 \mu \mathrm{l}$ of reverse transcription mixture contained $1 \mu \mathrm{g}$ of DNase I pretreated total RNA, $0.75 \mu \mathrm{g}$ of oligo $\mathrm{d}$ (T) primer, $6 \mu \mathrm{l}$ of $5 \mathrm{x}$ RT buffer, $10 \mathrm{mM}$ dithiothreitol, $0.5 \mathrm{mM}$ deoxynucleotides, $50 \mathrm{U}$ of
RNase inhibitor, and $240 \mathrm{U}$ of reverse transcriptase (Invitrogen). The RT reaction was carried out at $40{ }^{\circ} \mathrm{C}$ for $70 \mathrm{~min}$ followed by heat inactivation at $95{ }^{\circ} \mathrm{C}$ for $3 \mathrm{~min}$. The tested genes and the internal control ( $\beta$-actin) were amplified by PCR using $2 \mu$ RT products from each sample in a $20 \mu \mathrm{l}$ reaction containing Taq polymerase (0.01 U.ml $\left.{ }^{-1}\right)$, dNTPs (100 mM), $\mathrm{MgCl}_{2}(1.5 \mathrm{mM})$ and buffer $(50 \mathrm{mM}$ Tris-HCl). PCR reactions consisted of a first denaturing cycle at $97{ }^{\circ} \mathrm{C}$ for 5 min, followed by a variable number of cycles of amplification, consisting of denaturation at $96{ }^{\circ} \mathrm{C}$ for $30 \mathrm{sec}$, annealing for $30 \mathrm{sec}$, and extension at $72{ }^{\circ} \mathrm{C}$ for $1 \mathrm{~min}$. A final extension cycle of $72{ }^{\circ} \mathrm{C}$ for $15 \mathrm{~min}$ was included. Annealing temperature was adjusted for each target: $60{ }^{\circ} \mathrm{C}$ for $\mathrm{p} 53$, and 55 ${ }^{\circ} \mathrm{C}$ for Bax and $\beta$-actin. A control reaction without reverse transcriptase was included for every sample of RNA isolated to verify the absence of contamination. PCR products $(10 \mu \mathrm{l})$ were electrophoresed on 2\% agarose gels containing 100 ng. $\mathrm{ml}^{-1}$ ethidium bromide, and photographed with a Polaroid camera under ultraviolet illumination. Gel images were scanned, and the bands for Bax, $\mathrm{p} 53$, and $\beta$-actin were quantified using the myImageAnalysis Software, Thermo Scientific, USA ${ }^{33}$. Bax and p53 intensities were normalized to those of the corresponding $\beta$-actin band intensity for each sample.

\section{Statistical analysis}

Statistical analyses were performed using the GraphPad Prism 6 statistical software package (GraphPad Software Inc., La Jolla, CA, USA). Data are expressed as means with their standard Deviation (means \pm SD). Normality and homogeneity of the data were confirmed before 
ANOVA, and differences among the experimental groups were assessed by One Way ANOVA followed by post hoc Tukey's test. Values were considered significantly different at $\mathrm{p}<0.05$.

\section{RESULTS}

\section{Macroscopic and acidity changes in the} stomach:

There were no gastric lesions or haemorrhage in the stomachs of both the control and resveratrol (RES) treated groups of animals (Fig. 1 upper panel A \& B). On the contrary, chronic unpredictable stress (CUS) caused severe gastric mucosal damage characterized by observable mucosal lesions, including petechial lesions and haemorrhage bands of different sizes (Fig. 1 upper panel C). The CUS rats have a remarkably high ulcer index (UI), with a concomitant decrease in the gastric juice $\mathrm{pH}$ of more than $55 \%$ when compared to gastric juice $\mathrm{pH}$ of control or REStreated groups (Fig. 1 lower panel A \& B). Pretreatment with RES offered a significant protection against CUS-induced gastric lesions as apparent in the experimental rats that received RES as a concomitant drug with the stress protocol (Fig. 1 upper panel D), and the high percentage of protection by RES was associated with a restoration of normal gastric juice $\mathrm{pH}$ levels (Fig. 1 lower panel A \& B).

\section{Microscopic histopathological changes in the}

\section{stomach:}

Gastric histological sections stained with haematoxylin and eosin from the control group (Fig. 2 A \& $\mathrm{A}^{\prime}$ ) and RES treated control group (Fig. 2 B, \& B') showed normal architecture and revealed no gastric mucosa injuries. Conversely,
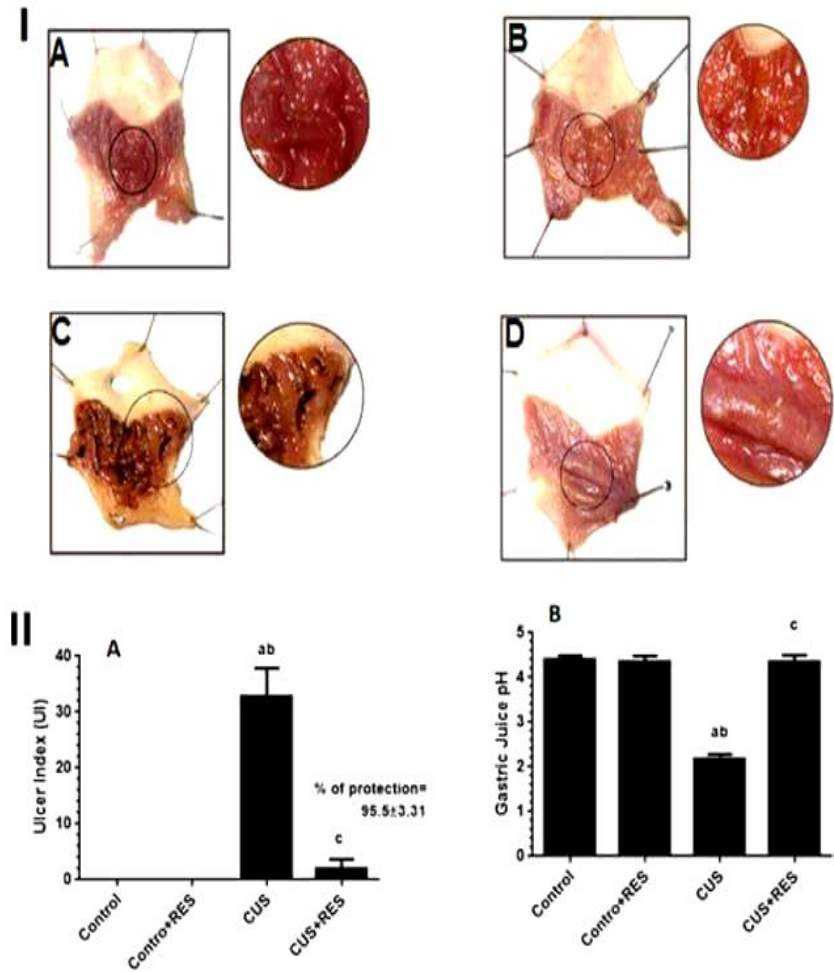

Figure (1). Assessment of gastric lesions in all experimental groups. Upper panel (I): The morphological changes in stomachs of all groups of rats showing large hemorrhagic ulcerated areas of different lengths in CUS group of rats (C), and absence of lesions areas in the control (A), Control+ RES (B), and CUS+RES (D) rats. Lower panel (II): The numerical values of gastric ulcer index (A), and gastric juice $\mathrm{pH}(\mathrm{B})$ in all groups of rats. Values are expressed as mean $\pm \mathrm{SD}(n=8$ in each group). (a) Significantly different compared to Control group. (b) Significantly different compared to Control+RES treated group. (c) Significantly different when to CUS group. Analysis was done by One Way ANOVA with post hoc Tukey's Test, and values were considered significantly different at $p<0.05$. CUS: Chronic unpredictable stress. RES: Resveratrol. UI: Ulcer index.

Conversely, sections obtained from CUS rats showed severe mucosal erosions and disruption of the surface epithelium, with necrotic lesions penetrating deeply into mucosa and reaching the muscularis mucosa, as well as extensive edema and leucocytes infiltration of submucosal layer (Fig. $2 \mathrm{C} \& \mathrm{C}^{\prime}$ ). Normal gastric architecture was superbly preserved in the CUS rats that were administered with RES(Fig.2D\&D'). 

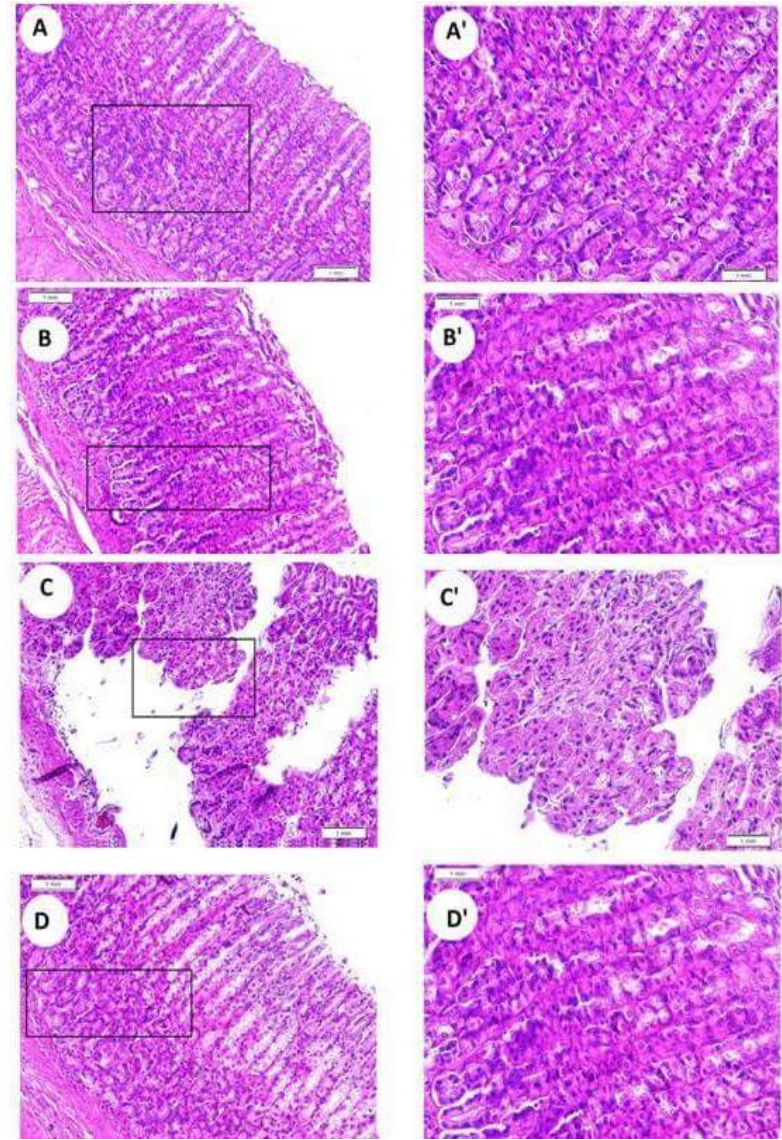

Figure (2). Photomicrographs of gastric tissues obtained from all experimental groups (HE stain, left panel at x200 and right panel at $\mathrm{x} 400$ of the square area of the corresponding tissue). The control group (A) and the control+RES group (B) show normal gastric architecture with gastric pits and gastric glands and no injuries to the gastric mucosa are seen. On the contrary, the CUS group (C) shows severe mucosal erosion and sever disruption of the surface epithelium with apoptotic and necrotic lesions penetrating deeply into mucosa, as well as extensive submucosal oedema and leucocytes infiltration. The CUS+RES group (D) remarkably shows normal architectures of gastric mucosa with intact layers and no pathologies of the surface epithelium or the submucosal tissues. HE: Haematoxylin and eosin. CUS: Chronic unpredictable stress. RES: Resveratrol.

\section{Gastric oxidative stress and nitrosative stress}

parameters:

Significant decrease in the levels of thiobarbituric acid reactive substances (TBARS), with a concomitant increase in the activity of superoxide dismutase (SOD), and no change in the levels of GPx were observed in the RES treated group as compared to control group. However, in the CUS group, a substantial significant 2.5 folds increase in the levels of TBARS, and significant decrease in both SOD and GPx activities were detected, indicating increased gastric lipid peroxidation and oxidative stress during CUS. The levels of TBARS, SOD activity, and GPx activity returned to their normal control levels when the CUS rats were treated with RES, revealing a potent antioxidant activity of RES (Fig. 3). On the other hand, the results did not show any significant changes, between all of the four experimental groups of rats, in the levels of inducible nitric oxide synthase (iNOS), nitrate, nitrite, or the nitrate/nitrite ratio.
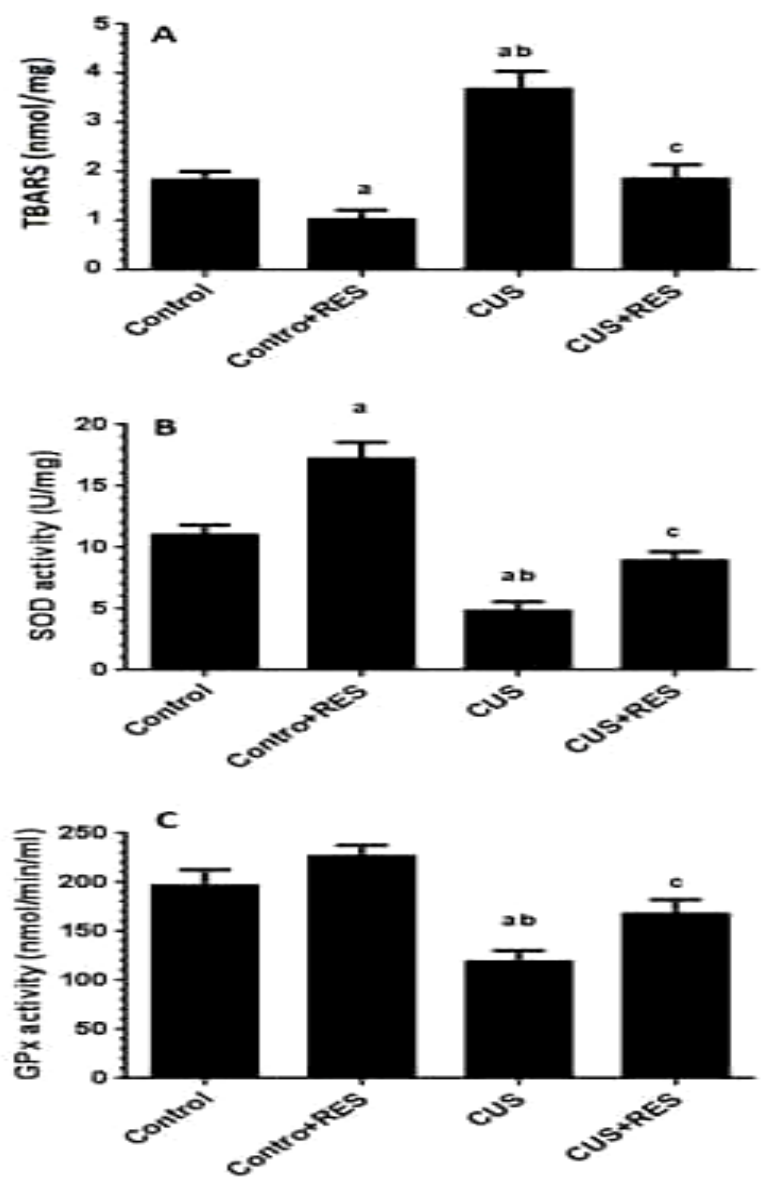

Figure (3). Levels of thiobarbituric acid reactive substances (TBARS; A), activity of superoxide dismutase (SOD; B), and activity of glutathione peroxidase (GPx; C) in the gastric tissue homogenates of all experimental groups of rats. Values are expressed as mean $\pm \mathrm{SD}$ ( $n=8$ in each group). (a) Significantly different compared to Control group. (b) Significantly different compared to Control+RES treated group. (c) Significantly different when to CUS group. Analysis was done by One Way ANOVA with post hoc Tukey's Test, and values were considered significantly different at $p<0.05$. CUS: Chronic unpredictable stress. RES: Resveratrol. 


\section{Gastric inflammatory markers:}

As shown in Figure 4, no significant change in the gastric tissue homogenates levels of interleukin-6 (IL-6) but significant decrease in the levels of tumor necrosis factor alpha (TNF- $\alpha)$ were seen in RES treated rats compared to control group. Considerable significant elevations of TNF- $\alpha$ and IL-6 levels were detected in the CUS group by 93 $\%$ and $116 \%$, respectively. On the other hand, significant decreases in the gastric levels of TNF- $\alpha$ and IL- 6 were seen in the CUS rats treated with RES compared with CUS group. The percentage improvements in the levels of IL- 6 and TNF- $\alpha$ in this CUS-RES groups of rats were $92 \%$ and $95 \%$, respectively, demonstrating the anti-inflammatory effect of RES (Fig. 4 A\&B). Indeed, resveratrol administration to control rats (control+RES) did not alter the levels of prostaglandin E2 (PGE2) as compared to control group. However, the synthesis of mucosal PGE2 was markedly (48 \%) suppressed in rats challenged with CUS, compared to the normal control rats. Adequately, contemporaneous treatment of rats with RES during CUS resulted in $84 \%$ improvement in the levels of PGE2 as compared to CUS rats (Fig. 4 C).

\section{Gastric levels of Bcl-2 and caspase 3 proteins}

The levels of the apoptosis executioner protein caspase 3 did not show any noteworthy change between the control and the RES treated control groups (Fig. 5 B). Interestingly, RES administration to control rats significantly enhanced the levels of the anti-apoptotioc protein Bcl-2 (Fig. 5 A). On the other hand, the levels of Bcl-2 were significantly (61\%) lowered and the levels of caspase 3 were tremendously (205\%) elevated in the gastric homogenates of CUS rats as compared to control rats, endorsing CUS-provoked apoptosis (Fig. 5 A \& B).
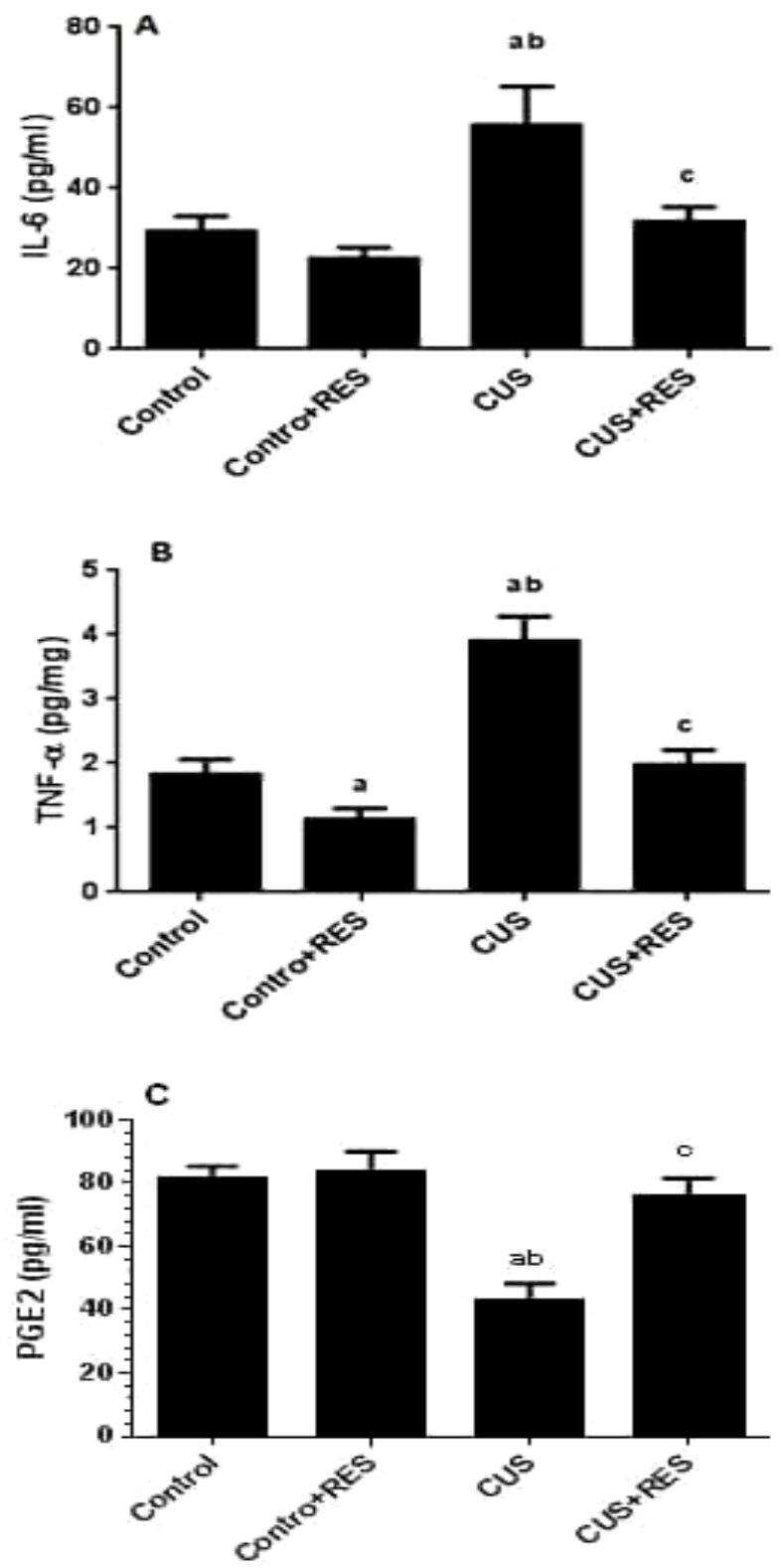

Figure (4). Levels of interleukin 6 (IL-6; A), tumor necrosis factor- $\alpha$ (TNF- $\alpha$; B), and prostaglandin E2 (PGE2; C) in the gastric tissue homogenates of all experimental groups of rats. Values are expressed as mean $\pm \mathrm{SD}$ ( $n=8$ in each group). (a) Significantly different compared to Control group. (b) Significantly different compared to Control+RES treated group. (c) Significantly different when to CUS group. Analysis was done by One Way ANOVA with post hoc Tukey's Test, and values were considered significantly different at $p<0.05$. CUS: Chronic unpredictable stress. RES: Resveratrol. 
Co-administration of RES during CUS ameliorated the CUS-provoked apoptosis and normalized the levels of both proteins to their control values (Fig.

\section{A \& B).}
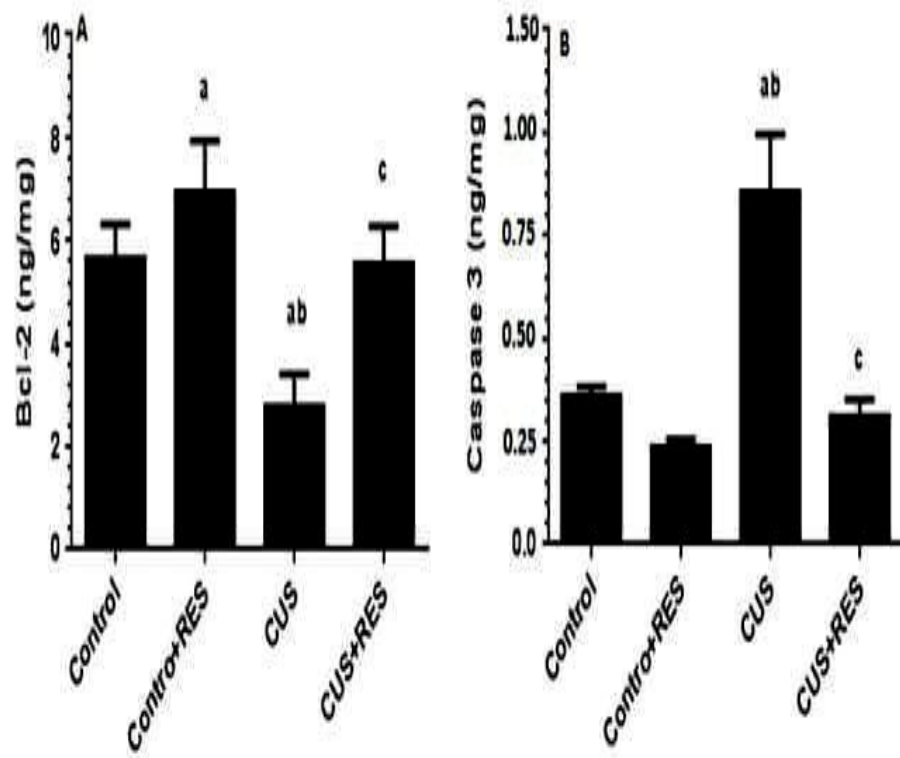

Figure (5). Levels of Bcl-2 (A) and caspase 3 (B) in the gastric tissue homogenates of all experimental groups of rats. Values are expressed as mean $\pm \mathrm{SD}$ ( $n=8$ in each group). (a) Significantly different compared to Control group. (b) Significantly different compared to Control+RES treated group. (c) Significantly different when to CUS group. Analysis was done by One Way ANOVA with post hoc Tukey's Test, and values were considered significantly different at $p<0.05$. CUS: Chronic unpredictable stress. RES: Resveratrol.

\section{Gastric levels of p53 mRNA and Bax mRNA:}

Figure 6 shows the transcriptional changes of gastric tissue p53 and Bax mRNAs in all groups of rats. All tested transcripts were detected, and RTPCR resulted in fragments similar in size to those expected. The levels of the $\beta$-actin transcript remained reasonably constant in all groups. In the control groups of rats that received the vehicle or RES, p53 mRNA and Bax mRNA were barely detectable and have similar level of expressions, correspondingly. However, it is noteworthy that the levels p53 mRNA and Bax mRNA increased several folds in the CUS rats. The Bax mRNA but not the P53 mRNA was significantly reduced upon treatment of CUS rats with RES, nevertheless both of them remained higher than those expressed in the control group (Fig. 6).
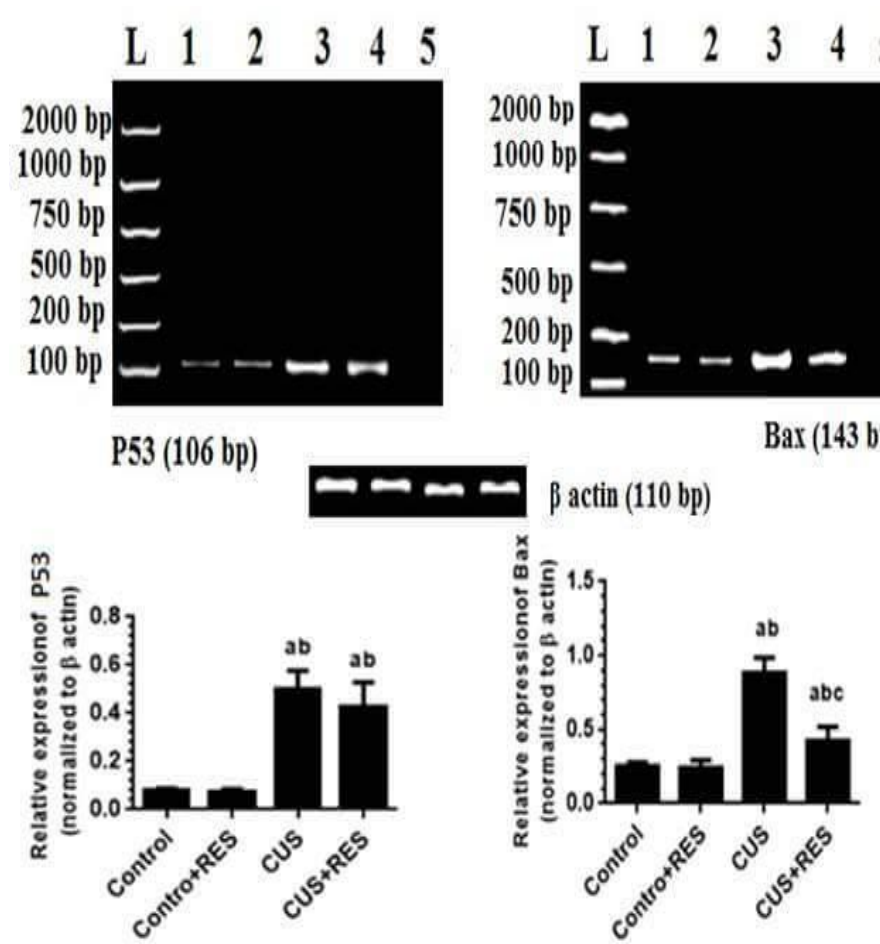

Figure (6). Semiquantitative reverse transcription PCR products and relative expression of gastric tissue mRNA of p53 and Bax in reference to $\beta$ actin mRNA (housekeeping gene). The RT-PCR products obtained from all groups were separated by $2 \%$ agarose gel electrophoresis with $100 \mathrm{ng}^{\mathrm{ml}}{ }^{-1}$ ethidium bromide. (L) Ladder, (1) Control, (2) Control+ RES, (3) CUS, (4) CUS+RES, and (5) Negative control in which reverse transcriptase was omitted. The relative density of images, normalized to $\beta$ actin, were analyzed by myImageAnalysis Software, Thermo Scientific, USA. Values are expressed as mean $\pm \mathrm{SD}$ ( $n=8$ in each group). (a) Significantly different compared to Control group. (b) Significantly different compared to Control+RES treated group. (c) Significantly different when to CUS group. Analysis was done by One Way ANOVA with post hoc Tukey's Test, and values were considered significantly different at $p<0.05$. CUS: Chronic unpredictable stress. RES: Resveratrol.

\section{DISCUSSION}

This study used the chronic unpredictable stress (CUS) protocol, which has been widely used in small animals to induced gastric ulcer as a justifiable paradigm to demonstrate similar 
mechanisms of stress-induced gastric injury like that of human. Supporting the stress-induced gastric injury previously reported in literature ${ }^{1,11 \text {, }}$ ${ }^{12}$, a major outcome of the current study showed that CUS for consecutive 21 days induced severe gastric lesions in rats as manifested by the microscopic and macroscopic evidences. The fundamental mechanisms by which CUS induced gastric lesions in this study are increased lipid peroxidation, oxidative stress, acid secretion and inflammation with a concomitant enhancement of gastric apoptosis and necrosis. The unique outcome of the current study is the first experimental demonstration of a complete remedy of CUS-induced gastric lesions by intraperitoneal administration of Resveratrol (RES) via reversing the CUS-triggered pathways that induced ulcer.

In the pathogenesis of gastric ulcers, the increase in gastric acidity and hydrogen ions are considered the most aggressive factors involved in CUS-induced ulcer ${ }^{34}$. In the present study, significant decrease in $\mathrm{pH}$ value of gastric juice was observed in stressed rats as compared to control rats. This increase in gastric acidity is undoubtedly due to increased production of hydrochloric acid $(\mathrm{HCl})$. During chronic stress, several mechanisms such as over secretion of histamine and stimulation of the stomach acid secretion both sympathetically and parasympathetically were attributed to contribute in increasing secretion of $\mathrm{HCl}^{35}$. Indeed, central sympathetic activation is invariably associated with stress ${ }^{36}$.

Prostaglandin E2 (PGE2) was shown to have protective effects against various gastric injury models ${ }^{37}$. Previous reports have shown that PGE2 is capable to maintain mucosal blood flow, stimulate mucus and bicarbonate secretion, improve the resistance of epithelial cells to injury induced by cytotoxins, and inhibit leukocyte recruitment 38,39 . In the current study, the significant decreased measured levels of PGE2 during chronic stress suggest the inhibitory roles of CUS on PGE2 synthesis and hence concealing its entire antiulcer protective action, and eventually provoking gastric ulcer. Moreover, and parallel to decreased PGE2 levels, activation of sympathetic nervous system by stress is usually associated with arteriolar vasoconstriction, thus reducing blood flow to the stomach, while activation of parasympathetic nervous system enhances gastric motility and muscular contraction leading to vascular compression with consequent mucosal ischemia ${ }^{40}$. Consequently, in both cases and following the ischemic event, superoxide anion $\left(\mathrm{O}_{2}^{-}\right)$leakage from mitochondrial electron transport chain is triggered, which further augments hydrogen peroxide $\left(\mathrm{H}_{2} \mathrm{O}_{2}\right)$ and hydroxyl radical $\left(\mathrm{OH}^{-}\right)$production and hence increasing tissue oxidative stress. Overproduction of reactive oxygen species (ROS) results in oxidative damage, including lipid peroxidation, protein oxidation, and DNA damage, which can lead to cell death ${ }^{41}$. Indeed, oxidative stress resulting in these mechanisms in the gastric mucosa has proved to be an important element in the development and progression of epithelial necrosis and mucosal ulceration ${ }^{42}$.

Gastric mucosal inflammation is too implicated to be involved in the pathogenesis of various gastric mucosal injuries ${ }^{43}$. It has been 
reported that overexpression of interleukin-6 (IL6) and tumor necrosis factor alpha (TNF- $\alpha$ ) are leading factors mediating neutrophil infiltration of gastric mucosa which further exacerbates ROS production ${ }^{44}$ through increased activity of neutrophils NADPH oxidase ${ }^{45}$. In agreement to this, a previous study showed positive correlation between the level of pro-inflammatory cytokines and ROS in a model of gastric ulcer and concluded that a decrease of ROS production caused decreased mucosal neutrophil infiltration and hence decrease ulcer index ${ }^{46}$. In agreement, the current study revealed increased levels of gastric IL-6, TNF- $\alpha$ and thiobarbituric acid reactive substances (TBARS) with a concomitant decrease in the activities of superoxide dismutase (SOD) and glutathione peroxidase (GPx). Taking together, the present study supports the suggestion that local gastric ROS generation could be an initiating or triggering event in the early phase of CUS-induced gastric mucosal injury ${ }^{47}$. On the other hand, previous studies documented that nitric oxide (NO) is considered as a gastroprotective factor, when released from gastric epithelium in smaller amounts ${ }^{48}$. In the stomach, NO is mainly produced by cNOS and iNOS enzymatic pathways 49. NO synthase and NO act to maintain mucosal integrity through modulating various functions such as mucus secretion, bicarbonate secretion, and mucosal blood flow ${ }^{50,51}$. NO has also been reported protect against gastric damage by promotion of PGE2 synthesis ${ }^{52}$. In the present study, CUS did not show any changes in the levels of iNOS or nitrate/nitrite levels, indicating that the decrease in the levels of PGE2 or the increase in ROS occurs independently of NO.
The current study is showing, for the first time, that CUS induced both apoptosis and necrosis in rat's gastric tissue as approved by the molecular and histopathological evidences. This novel finding has shown the activation of intrinsic apoptotic pathway to be a common death pathway of the gastric mucosa cells in CUS. In general, two major pathways can induce apoptotic cell death; the intrinsic, mitochondrial, pathway involves Bax/Bcl-2, cytochrome $\mathrm{C}$ and caspase-9, and the extrinsic, death receptor, pathway is involved Fas and caspase- 8 protein. Both pathways converge to a final apoptosis execution step which includes caspase 3. Caspase 3 is considered to be a major executioner protease which is essential for apoptotic death in mammalian cells ${ }^{53}$. On the other hand, p53 is a tumor suppressor protein which can activate both extrinsic and intrinsic pathways of apoptosis ${ }^{54}$. It has been reported that when there is an irreparable and severe DNA damage, p53 induces the expression of $\mathrm{Bcl}-2$ pro-apoptotic family members: Bax, Puma and Noxa, resulting in shifting of the balance towards intrinsic proapoptotic effects ${ }^{55}$. In the current study, CUS resulted in a significant increase in the gastric caspase 3 levels and enhanced mRNA levels of P53 and Bax genes. Also, there was a significant decrease in the gastric levels of Bcl-2. Although, These finding suggests an CUS activation of the intrinsic pathway of apoptosis, the components of the extrinsic pathway activated by TNF- $\alpha$ could be also involved in this apoptotic pathway and further studies is required. However, as noticed in the above discussion, the diversity of different pathways underlying stress-induced gastric ulcer, and the complex nature of pathways participating in healing always make the treatment a 
complicated challenge ${ }^{56}$. It is well accepted that maintaining equilibrium between aggressive and defensive factors is always a critical objective in ulcer management. In the current study, RES intraperitoneal administration completely protected the gastric tissue from CUS-induced ulcer. The data shows complete reversal of all major pathways involved in CUS induced ulcer by RES via potent antioxidant, anti-inflammatory and interestingly, anti-apoptotic protective effects. Findings of the present study are similar to those obtained by previous research which showed that RES treatment supported antioxidant defences and to reduce oxidative gastric damage in cholestatic rats ${ }^{22}$. Additionally, RES were reported to have both protective and therapeutic effects on oxidative gastric damage by suppressing proinflammatory cascades, including the activation of pro-inflammatory cytokines, accumulation of neutrophils and release of ROS in acetic acid-induced gastric ulcer ${ }^{57}$. However, interestingly, the current study is the first that shows that RES protects against CUS-induced apoptosis. Given that normal levels of $\mathrm{Bcl}-2$ and caspase 3 and normal expression levels of both p53 and Bax in the gastric tissue of normal rats treated with RES, it seems that RES main mechanism of action is due to inhibition of oxidative stress-induced apoptosis rather than regulating the levels of these apoptotic and anti-apoptotic proteins per se. Indeed, It has been reported that high levels of ROS provoke p53 levels, and disrupt the cells inner and outer mitochondrial membranes, relocating Bax, and induce the release of the cytochrome c, and activates caspase 9 and 3 resulting in cell apoptosis ${ }^{58}$.
However, the current data are not in complete agreement with those reported by Brzozowski and colleagues ${ }^{49,50}$ who showed that RES treatment delay healing of gastric ulcer induced by ischemiareperfusion and acetic acid by reducing PGE2 synthesis via specific inhibition of cyclooxygenase (COX)-1. In those studies, RES treatments were applied intragastrically, which may be the reason for the lack of its facilitatory effect on the healing of gastric ulcer as observed in the present study were RES treatments were given intraperitoneally. Supporting to this, unlike Brzozowski studies, RES did not affect PGE2 levels in the control rats and significantly decreased its levels in the ulcer group of rats.

In conclusion, the present study investigated the gastric mucosal damage in a rat model of chronic unpredictable stress (CUS), and shows that supplementation with the polyphenol resveratrol successfully offers a protective effect against CUS-induced gastric ulcer. The mechanisms of resveratrol ameliorative action entail potent antioxidant, anti-inflammatory, and anti-apoptotic effects.

\section{ACKNOWLEDGMENTS}

The author sincerely thanks Mr Mahmoud AlKhateeb from the Department of Physiology and Mr Riyadh Al-Eissa from the Department of Biochemistry, College of Medicine, King Khalid University for valuable technical assistance in the experimental work. Thanks are due to Professor Samir Zaki from the Histology Division, Department of Anatomy, College of Medicine, King Khalid University for help with the histological studies. Preliminary results of this 
study were presented previously in an abstract form by the author, as a recipient of the American Physiological Society Travel Award, at the First Pan American Congress of Physiological Sciences that was hosted by the Brazilian Physiological Society in Foz do Iguassu, Parana, Brazil ${ }^{59}$.

\section{CONFLIT OF INTEREST}

The author has no conflict of interest to declare.

\section{REFERENCES}

1. Guo S, Qian G, Qing J, Wei H, Xue G, Ji-Min C. Gastric mucosal damage in water immersion stress: Mechanism and prevention with GHRP-6. World J Gastroenterol. 18(24): 3145-3155, 2012.

2. Roy MP, Kirschbaum C, Steptoe A. Psychological, cardiovascular, and metabolic correlates of individual differences in cortisol stress recovery in young men. Psychoneuroendocrinol. 26: 375-391, 2001.

3. Pruett SB. Quantitative aspects of stress-induced immunomodulation. Int. Immunopharmacol. 1:507-520, 2001.

4. Fitzpatrick F, Christeff N, Durant S, Dardenne M, Nunez EA, Homo-Delarche F. Glucocorticoids in the non-obese diabetic (NOD) mouse: Basal serum levels, effect of endocrine manipulation and immobilization stress. Life Sci. 50: 1063-1069, 1992.

5. Dobson H, Smith RF. What is stress, and how does it affect reproduction? Anim Reprod Sci. 6061: 743-752, 2000.

6. Gareri P, Falconi U, De Fazio P, De Sarro G. Conventionaland new antidepressant drugs in the elderly. Prog Neurobiol. 61(4): 353-396, 2000.

7. Cook DJ, Griffith LE, Walter SD, Guyatt GH, Meade MO, Heyland DK, Kirby A, Tryba M. The attributable mortality and length of intensive care unit stay of clinically important gastrointestinal bleeding in critically ill patients. Crit Care. 5(6): 368-375, 2001.

8. Cook DJ, Fuller HD, Guyatt GH, Marshall JC, Leasa D, Hall R, Winton TL, Rutledge F, Todd T, Roy P, Lacroix J, Griffith L, Willan A. Risk factors for gastrointestinal bleeding in critically ill patients. N Engl J Med. 330(6): 377-381, 1994.

9. Ackerman SH, Hofer MA, Weiner H. Age at maternal separation and gastric erosion susceptibility in the rat. Psychosom Med. 37: 180184, 1975.

10. Hamaguchi M, Watanabe $\mathbf{T}$, Higuchi $\mathbf{K}$, Tominaga K, Fujiwara Y, Arakawa T. Mechanisms and roles of neutrophil infiltration in stress-induced gastric injury in rats. Dig Dis Sci. 46(12): 2708-2015, 2001.

11. Kamper EF, Chatzigeorgiou A, Tsimpoukidi O, Kamper M, Dalla C, Pitychoutis PM, Papadopoulou-Daifoti Z. Sex differences in oxidant/antioxidant balance under a chronic mild stress regime. Physiol Behav. 98: 215-222, 2009.

12. Lucca G, Comim CM, Valvassori SS, Réus GZ, Vuolo F, Petronilho F, Gavioli EC, Dal-Pizzol F, Quevedo J. Increased oxidative stress in submitochondrial particles into the brain of rats submitted to the chronic mild stress paradigm. $J$ Psychiatr Res. 43(9): 864-869, 2009.

13. Slomiany BL, Piotrowski J, Slomiany A. Downregulation of endothelin-1 by interleukin-4 during gastric ulcer healing. Biochem Biophys Res Commun. 263(2): 591-595, 1999.

14. Nassar NN, Schaalan MF, Zaki HF, Abdallah DM. Octreotide ameliorates gastric lesions in chronically mild stressed rats. World $J$ Gastroenterol. 17(9): 1135-1142, 2011.

15. Ribeiro JM, Lucas M, Baptista A, Victorino RM. Fatal hepatitis associated with ranitidine. $A m$ J Gastroenterol. 95(2): 559-560, 2000. 
16. Schmeda-Hirschmann G, Yesilada E. Traditional medicine and gastroprotective crude drugs. J Ethnopharmacol. 100(1-2): 61-66, 2005.

17. Soleas GJ, Diamandis EP, Goldberg DM. The world of resveratrol. Adv Exp Med Biol. 492: 159182,2001

18. Holme AL, Pervaiz S. Resveratrol in cell fate decisions. J Bioenerg Biomembr. 39(1): 59-63, 2007.

19. Seifried HE, Anderson DE, Fisher EI, Milner JA. A review of the interaction among dietary antioxidants and reactive oxygen species. J Nutr Biochem. 18(9): 567-579, 2007.

20. Pirola L, Fröjdö S. Resveratrol: One molecule, many targets. IUBMB Life. 60(5): 323-332, 2008.

21. Giovannini C, Filesi C, D'Archivio M, Scazzocchio B, Santangelo C, Masella R. Polyphenols and endogenous antioxidant defences: effects on glutathione and glutathione related enzymes. Ann Ist Super Sanita. 42(3): 336347, 2006.

22. Kirimlioglu V, Ara C, Yilmaz M, Ozgor D, Isik B, Sogutlu G, Kirimlioglu H, Karabulut AB, Yilmaz S, Kayaalp C, Yologlu S. Resveratrol, a red wine constituent polyphenol, protects gastric tissue against the oxidative stress in cholestatic rats. Dig Dis Sci. 51(2): 298-302, 2006.

23. National Research Council (US) Committee. Guide for the Care and Use of Laboratory Animals. $8^{\text {th }}$ ed., National Academies Press, Washington DC, 2011.

24. Eleawa SM, Alkhateeb MA, Alhashem FH, BinJaliah I, Sakr HF, Elrefaey HM, Elkarib AO, Alessa RM, Haidara MA, Shatoor AS, Khalil MA. Resveratrol reverses cadmium chlorideinduced testicular damage and subfertility by downregulating p53 and Bax and upregulating of gonadotropins and $\mathrm{Bcl}-2$ gene expression. $J$ Reprod Dev. 60(2): 115-127, 2014.
25. Harro J, Häidkind R, Harro M, Modiri AR, Gillberg PG, Pähkla R, Matto V, Oreland L. Chronic mild unpredictable stress after noradrenergic denervation: Attenuation of behavioural and biochemical effects of DSP-4 treatment. Eur Neuropsychopharmacol. 10(1): 5$16,1999$.

26. Stroff T, Plate S, Ebrahim JS, Ehrlich KH, Respondek M, Peskar BM. Tachykinin-induced increase in gastric mucosal resistance: role of primary afferent neurons, CGRP and NO. Am J Physiol. 271: G1017-G1027, 1996.

27. Peskar BM, Ehrlich K, Peskar BA. Role of ATP-sensitive potassium channels in prostaglandin-mediated gastroprotection in the rat J Pharmacol Exp Ther. 301(3): 969-974, 2002.

28. Hano J, Bugajski J, Danek L. Effect of adrenergic blockade on gastric secretion altered by catecholamines in rats. Arch Immunol Ther Exp (Warsz). 24(4): 507-524, 1976.

29. Tan PV, Nyasse B, Dimo T, Mezui C. Gastric cytoprotective anti-ulcer effects of the leaf methanol extract of Ocimum suave (Lamiaceae) in rats. J Ethnopharmacol. 82(2-3): 69-74, 2002.

30. Ketuly KA, Abdulla MA, Hadi HA, Mariod AA, Abdel-Wahab SI. Anti-ulcer activity of the 9alpha-bromo analogue of Beclomethasone dipropionate against ethanol-induced gastric mucosal injury in rats. J Med Plants Res. 5(4): 514-520, 2011

31. Feng Y, Shi Z, Fang $\mathbf{X}$, Xu M, Dai J. Perfluorononanoic acid induces apoptosis involving the Fas death receptor signaling pathway in rat testis. Toxicol Lett. 190(2): 224230, 2009.

32. Li GY, Xie P, Li HY, Hao L, Xiong Q, Qiu T. Involment of $\mathrm{p} 53, \mathrm{Bax}$, and $\mathrm{Bcl}-2$ pathway in microcystins-induced apoptosis in rat testis. Environ Toxicol. 26(2(: 111-117, 2011. 
33. Szafran AT, Mancini MA. The myImageAnalysis project: A web-based application for high-content screening. Assay Drug Dev Technol. 12(1): 87-99, 2014.

34. Lüllmann H, Mohr K, Ziegler A, Bieger D. Color atlas of pharmacology. $2^{\text {nd }}$ ed., Thieme Press, Stuttgart, 2000, pp 166.

35. Grossman MI. Control of gastric secretion. In: Gastrointestinal disease: Pathophysiology, diagnosis and management. MH Sleisenzer and JS Fordtran (eds), $2^{\text {nd }}$ ed., W B Saunders Co, Philadelphia, 1978, pp 640-659.

36. Corrodi H, Fuxe K, Hökfelt T. The effect of immobilization stress on the activity of central monoamine neurons. Life Sci. 7(1): 107-112, 1968.

37. Brzozowski T, Konturek PC, Konturek SJ, Brzozowska I, Pawlik T. Role of prostaglandins in gastroprotection and gastric adaptation. $J$ Physiol Pharmacol. 56(5): 33-55, 2005.

38. Miller TA. Protective effects of prostaglandins against gastric mucosal damage: Current knowledge and proposed mechanisms. Am $J$ Physiol. 245(5): G601-G623, 1983.

39. Ishihara K, Kuwata H, Ohara S, Okabe H, Hotta K. Changes of rat gastric mucus glycoproteins in cytoprotection: Influences of prostaglandin derivatives. Digestion. 39(3): 162$171,1988$.

40. Ito M, Shichijo K, Sekine I. Gastric motility and ischemic changes in occurrence of linear ulcer formation induced by restraint-water immersion stress in rat. Gastroenterol Jpn. 28(3): 367-373, 1993.

41. Das D, Bandyopadhyay D, Bhattacharjee M, Banerjee RK. Hydroxyl radical is the major causative factor in stress-induced gastric ulceration. Free Radic Biol Med. 23(1): 8-18, 1997.
42. Shian WM, Sasaki I, Kamiyama Y, Naito H, Matsuno S, Miyazawa T. The role of lipid peroxidation on gastric mucosal lesions induced by water-immersion-restraint stress in rats. Surg Today. 30(1): 49-53, 2000.

43. Odashima M, Otaka M, Jin M, Komatsu K, Wada I, Horikawa Y, Matsuhashi T, Hatakeyama N, Oyake J, Ohba R, Watanabe S, Linden J. Attenuation of gastric mucosal inflammation induced by aspirin through activation of A2Aadenosine receptor in rats. World J Gastroenterol. 12(4): 568-573, 2006.

44. Yoshikawa T, Naito Y. The role of neutrophils and inflammation in gastric mucosal injury. Free Radic Res. 33(6): 785-794, 2000.

45. Nakagiri A, Murakami M. Roles of NADPH oxidase in occurrence of gastric damage and expression ofcyclooxygenase-2 during ischemia/reperfusion in rat stomachs. J Pharmacol Sci. 111(4): 352-360, 2009.

46. Konturek PC, Duda A, Brzozowski T, Konturek SJ, Kwiecien S, Drozdowicz D, Pajdo R, Meixner H, Hahn EG. Activation of genes for superoxide dismutase, interleukin-1beta, tumor necrosis factor-alpha, and intercellular adhesion molecule-1 during healing of ischemiareperfusion-induced gastric injury. Scand J Gastroenterol. 35(5): 452-463, 2000.

47. Hildeman DA, Mitchell T, Kappler J, Marrack P. T cell apoptosis and reactive oxygen species. $J$ Clin Invest. 111(5): 575-581, 2003.

48. Kwiecień S, Brzozowski T, Konturek PCh, Konturek SJ. The role of reactive oxygen species in action of nitric oxide-donors on stress-induced gastric mucosal lesions. J Physiol Pharmacol. 53(4-2): 761-773, 2002.

49. Brzozowski T, Konturek PC, Pajdo R, PtakBelowska A, Kwiecien S, Pawlik M, Drozdowicz D, Sliwowski Z, Brzozowski B, Konturek SJ, Pawlik WW. Physiological 
mediators in nonsteroidal anti-inflammatory drugs (NSAIDs)-induced impairment of gastric mucosal defense and adaptation. Focus on nitric oxide and lipoxins. J Physiol Pharmacol. 59(2): 89-102, 2008.

50. Brzozowski T, Kwiecień S, Konturek PC, Konturek SJ, Mitis-Musiol M, Duda A, Bielański W, Hahn EG. Comparison of nitric oxide-releasing NSAID and vitamin $\mathrm{C}$ with classic NSAID in healing of chronic gastric ulcers; involvement of reactive oxygen species. Med Sci Monit. 7(4): 592-599, 2001.

51. Brzozowski T, Konturek P, Konturek SJ, Kwiecień S, Sliwowski Z, Pajdo R, Duda A, Ptak A, Hahn EG. Implications of reactive oxygen species and cytokines in gastroprotection against stress-induced gastric damage by nitric oxide releasing aspirin. Int $J$ Colorectal Dis. 18(4): 320-329, 2003.

52. Salvemini D, Misko TP, Masferrer JL, Seibert K, Currie MG, Needleman P. Nitric oxide activates cyclooxygenase enzymes. Proc Natl Acad Sci U S A. 90(15): 7240-7244, 1993.

53. Said TM, Paasch U, Glander HJ, Agarwal A. Role of caspases in male infertility. Hum Reprod Update. 10(1): 39-51, 2004.

54. Kroemer G, Galluzzi L, Brenner C. Mitochondrial membrane permeabilization in cell death. Physiol Rev. 87(1): 99-163, 2007.

55. Thornborrow EC, Patel S, Mastropietro AE, Schwartzfarb EM, Manfredi JJ. A conserved intronic response element mediates direct p53dependent transcriptional activation of both the human and murine bax genes. Oncogene. 21(7): 990-999, 2002.

56. El-Moselhy MA, Abdel-Hamid NM, AbdelRaheim SR. Gastroprotective effect of nicorandil in indomethacin and alcohol-induced acute ulcers. Appl Biochem Biotechnol. 152(3): 449-459, 2009.
57. Solmaz A, Sener G, Cetinel S, Yüksel M, Yeğen C, Yeğen BC. Protective and therapeutic effects of resveratrol on acetic acid-induced gastric ulcer. Free Radic Res. 43(6): 594-603, 2009.

58. Jia YT, Ma B, Wei W, Xu Y, Wang Y, Tang HT, Xia ZF. Sustained activation of nuclear factor-kappaB by reactive oxygen species is involved in the pathogenesis of stress-induced gastric damage in rats. Crit Care Med. 35(6): 1582-1591, 2007.

59. Bin-Jaliah I. Resveratrol effects on chronic unpredictable stress-induced gastric ulcer. Proc Pan Am Congr Physiol Sci. 1: 242, 2014. 